Aim of the study: Sunitinib-related side effects may develop as a result of the pharmacokinetic pathway affects the of the drug.

Material and methods: Data on mRCC patients were obtained from the hospital archives. Outcomes of patients were evaluated in terms of related prognostic factors, sunitinib adverse events during the treatment, and two different sunitinib dosing schedules.

Results: Seventy patients diagnosed with $\mathrm{mRCC}$ and treated with sunitinib were analyzed for prognostic factors and survival rates. During the mean follow-up of 33.5 months, 38 (54\%) patients were alive and 32 (46\%) patients died. The median time of overall survival (OS) and progression-free survival (PFS) was 27 months (12-61) and 19 months (5-45), respectively. In univariate analysis, good prognostic risk group according to the Memorial Sloan-Kettering Cancer Center (MSK$\mathrm{CC}$ ), hypothyroidism as sunitinib toxicity and patients on sunitinib treatment more than 1 year were favorable prognostic factors for OS. Leukopenia and fatigue as sunitinib toxicity were poor prognostic factors for OS. PFS and OS of the patients were not significantly different when we compared intermittent (4/2) vs. continuous treatment dosing schedules. Conclusions: As a result of this trial, having hypothyroidism as an adverse effect of sunitinib was a favorable prognostic factor for OS and PFS in mRCC patients. It was also found that $4 / 2$ and continuous dosing schedules of sunitinib did not give rise to different outcomes in $\mathrm{MRCC}$ patients.

Key words: metastatic renal cell carcinoma, prognostic factors, dosing schedule, sunitinib toxicity.

Contemp Oncol (Pozn) 2016; 20 (2): 147-152 DOI: $10.5114 /$ wo.2016.60069

\section{Prognostic relevance of sunitinib toxicities and comparison of continuous vs. intermittent sunitinib dosing schedule in metastatic renal cell cancer patients}

Çetin Ordu ${ }^{1}$, Kezban N. Pilanci ${ }^{1}$, Nilüfer Avcl ${ }^{2}$, İbrahim Yıldız ${ }^{3}$, Gül Alço ${ }^{4}$ Özkan Demirhan ${ }^{1}$, Ülkühan I. Köksal ${ }^{1}$, Filiz Elbüken ${ }^{4}$, Coskun Tecimer ${ }^{1}$, Gökhan Demir ${ }^{1}$

${ }^{1}$ Istanbul Bilim University, Istanbul, Turkey

${ }^{2}$ Balikesir State Hospital, Istanbul, Turkey

${ }^{3}$ Istanbul University Oncology Institute, Balikesir, Turkey

${ }^{4}$ Gayrettepe Florence Nightingale Hospital, Istanbul, Turkey

\section{Introduction}

Renal cell carcinoma (RCC) constitutes only $3 \%$ of adult malignancies but is the sixth leading cause of cancer-related death due to difficulties of effective therapy for locally advanced and metastatic disease. The multitargeted tyrosine kinase inhibitor (TKI) sunitinib has emerged as one of the standards of care for favorable and intermediate-risk metastatic renal cell carcinoma (mRCC). Sunitinib selectively inhibits tyrosine kinase activity of the vascular endothelial growth factor (VEGF) receptor, the platelet-derived growth factor receptor (PDGFR), the stem cell factor receptor (the cytokine receptor c-Kit), the FMS-like tyrosine- kinase 3 receptor (FLT)-3, and glial cell line-derived neurotrophic factor receptor (rearranged during transfection; RET). This inhibits tumoral delivery of blood and nutrients required for growth, which ultimately leads to cancer cell death. Sunitinib also has a direct inhibitory effect on tumor cells [1].

The rate of sunitinib-related adverse events was $19-50 \%$ and the percentage of patients' required dose reduction was $8-30 \%$ in the pivotal firstline trial and another sunitinib open access program, respectively [2, 3]. As it is known, drug dose and treatment duration are directly correlated with response to treatment and survival [4]. According to baseline patient characteristics, the most widely used prognostic factor model is from the Memorial Sloan Kettering Cancer Center (MSKCC) [5]. To date only retrospective analyses about the association between occurrence of a defined adverse event and clinical outcome are available. Oncologists have had to adapt to manage novel side effects of targeted therapies, such as rash, hypertension, hypothyroidism, diarrhea and hematologic toxicity. Some toxicities are related to the "on-target" effects of the drug and its inhibition of the pathway. Such toxicities are termed mechanism-based toxicities (MBTs) [6]. Dose modifications should thus be based not only on toxicities but also on pharmacokinetic (PK) and pharmacodynamic (PD) variables [7].

Presence of MBT can be used as a predictive marker in diseases for which pathway inactivation is sufficient to determine clinical activity. Multiple early-phase clinical studies have shown that the development of on-target effects in normal tissue can be correlated with pathway inhibition in tumors. It is also critical to state that MBTs can only be used as predictors of outcome after initiating treatment. Thereby, they can be taken as surrogates for the further clinical benefit of patients who continue therapy [6]. 
In the literature, hypertension and hypothyroidism are the most studied sunitinib adverse effect as predictive factors for patient outcome [8-16]. Although further studies are required, the abnormalities in thyroid function or blood pressure following treatment with sunitinib may be potential biomarkers for tumor response to multitargeted agents.

Sunitinib has been studied in cancer patients using various schedules. The recommended dose of sunitinib was $50 \mathrm{mg}$ in a $4 / 2$ intermittent schedule using 4-week treatment followed by a 2 -week rest period in a phase 1 trial [17]. Intermittent dosing schedules were sequentially conducted in two open-label, single-arm, phase 2 trials, in patients with $\mathrm{mRCC}$ and disease progression while receiving first-line cytokine therapy $[18,19]$. Furthermore, in one of the phase 2 trials, the concentration of sunitinib and its active metabolite declined to pre-dose levels during the 14-day rest period, leading to potential lack of drug exposure during which tumors could potentially progress [20]. So, several considerations have led to the investigation of continuous dosing schedules.

The purpose of this study was to evaluate sunitinib's adverse effects on patient outcome and compare 4/2 vs. continuous dosing schedules of sunitinib in mRCC.

\section{Material and methods}

Patients with mRCC who were treated with sunitinib were included in the study between June 2007 and January 2012. The patients were classified according to the MSKCC risk score [18].

Two different schedules of sunitinib were used for management of mRCC. The four weeks with two weeks off schedule (4/2) included 4 weeks of $50 \mathrm{mg}$ /day, followed by a 2 -week break. In the continuous dosing schedule, patients were allowed to start with a daily dose of $37.5 \mathrm{mg}$

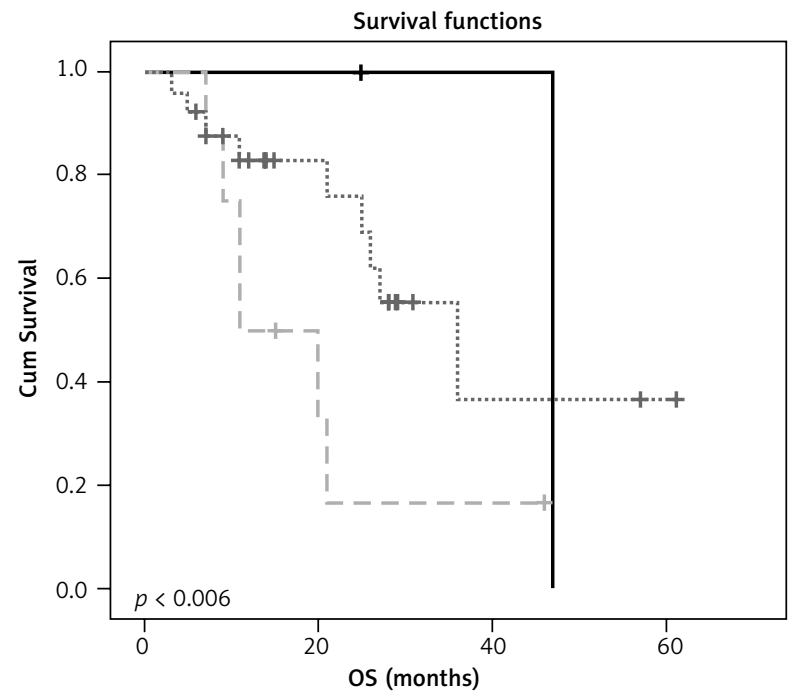

Kaplan-Meier curve of overall survival analysis according to MSKCC risk groups

$$
\begin{aligned}
& \neg \text { good } \\
& \ldots . \cdots=\text { intermedia } \\
& { }_{-}=\text {poor }
\end{aligned}
$$

Fig. 1. The median OS of patients in good prognostic risk factor groups was significantly longer than that of patients in intermediate and poor prognostic groups (47 m vs. $36 \mathrm{~m}$ vs. $11 \mathrm{~m}, p=0.006$ ) of sunitinib. Patient follow-up was performed at least on a monthly basis during the treatment exposure. Hematological and non-hematological toxic effects were graded according to $\mathrm{NCl}-\mathrm{CTC}$ version 3.0 [21]. All 70 patients were evaluated anatomically and every three months.

Histologically or cytologically proven RCC, sunitinib-treated patients were included in the trial. Patients whose mRCC was managed with TKIs other than sunitinib and who had less than 12 months of follow-up were excluded from the trial. Between June 2007 and January 2012, a total of 70 metastatic mRCC patients were available from hospital center archives.

\section{Statistical analysis}

SPSS version 17 for Windows was used for the statistical analysis. The $\chi^{2}$ test was used to investigate the association between categorical outcomes. Survival differences were calculated using the Kaplan-Meier method. Overall survival (OS) and progression-free survival (PFS) were calculated from the beginning of sunitinib use. Univariate Cox regression models were used to evaluate the effect of each specific parameter. Multivariate Cox regression models were performed to identify the independent risk factors for survival. $P \leq 0.05$ was considered to be statistically significant.

\section{Results}

At a mean of 33.5 months follow-up, 38 patients (54\%) were alive and 32 patients (46\%) died. Median age was 64 (range 26-78). Median OS was 27.0 months (range 12-61) and median progression-free survival (PFS) was 19 months (range 5-45). Five (7\%) patients had non-clear cell histopathology. Sixteen patients (23\%) were in poor prognostic risk factor groups according to MSKCC. Median duration of sunitinib treatment was 12.1 months (range 5-36). The numbers of patients with metastasectomy and nephrectomy were 18 and 46 respectively. Percentages of metastatic lesion locations were lung (32), bone (22), lymph node (10), brain (10), liver (10), surrenal (8), and pancreas (4) respectively. Median number of metastatic organs was 2. Median OS and PFS of patients with metastasectomy were non-significantly longer than the patients without metastasectomy (47 vs. 26 months, $p=0.630$ for OS and 24 vs. 19 months, $p=0.334$ for PFS). In univariate analyses, being a good prognostic risk group according to MSKCC, hypothyroidism as sunitinib toxicity and patients on sunitinib treatment for more than 1 year were favorable prognostic factors for OS (Figs. 1-3). In multivariate analysis, good risk group was the independent favorable risk factor for OS according to MSKCC ( $p=$ 0.033, OR: 2.458, 95\% Cl: 1.078-5.606). On the other hand, patients with leukopenia and fatigue as side effects of sunitinib had significantly shorter OS than patients without those side effects (Table 1 ).

Eventually, 58 patients (83\%) needed a dose reduction because of treatment-related adverse events. Mucositis, fatigue, and a combination of grade $2-3$ adverse events were the most frequent reasons for dose reduction. The numbers of patients treated with the $4 / 2$ vs. continuous dosing schedule were 36 and 34 respectively. Patients' characteristics are summarized in Table 2. 


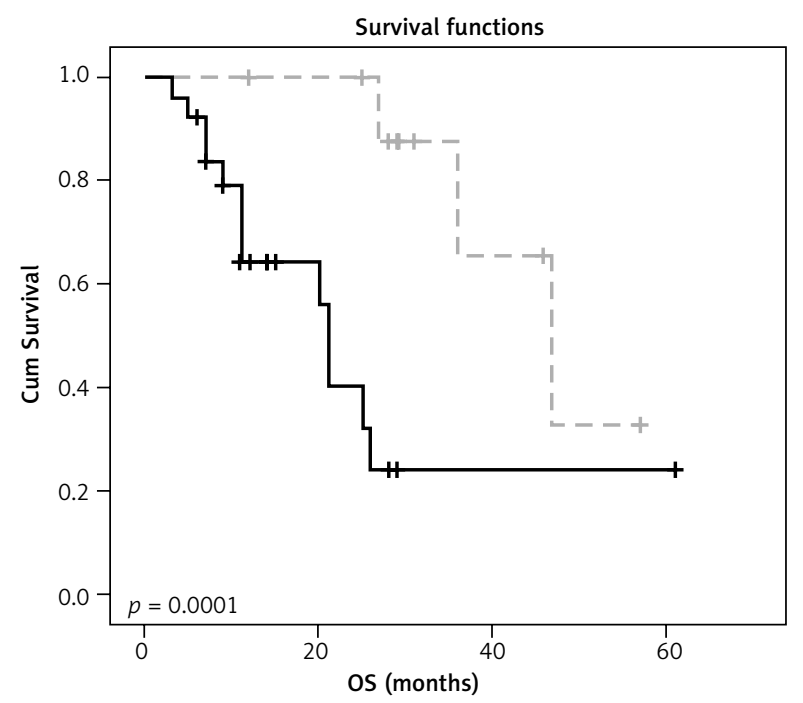

Kaplan-Meier curve of overall survival analysis according to sunitinib duration

$$
\begin{aligned}
& \Gamma>1 \text { year } \\
& -^{\mathrm{r}}=1 \text { year }
\end{aligned}
$$

Fig. 2. The median OS of patients who had sunitinib durations of more than 12 months was significantly longer compared to those who had sunitinib durations of less than 12 months. (47 m vs. $21 \mathrm{~m}$, $p<0.0001)$

There was no statistically significant difference in median OS and PFS of the patients in terms of treatment schedule (4/2 vs. continuous dosing schedule; Fig. 4). Except for leukopenia, no statistically significant discrepancy was found comparing the two treatment schedules, in terms of any kind of toxicity rates with sunitinib (Table 3 ).

\section{Discussion}

In this study, treatment-related adverse events were mostly grade 1 or 2 , and only a few grade 3 toxicities were

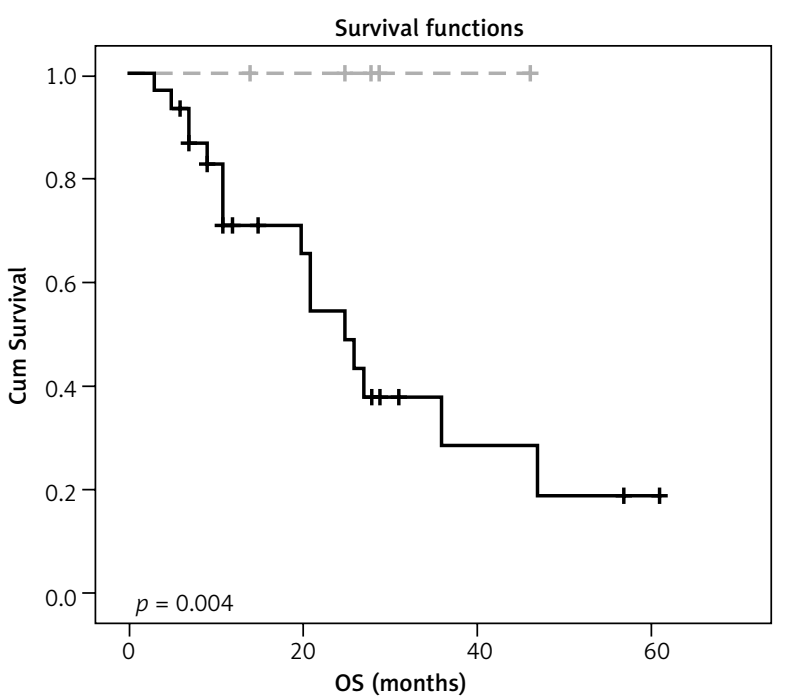

Kaplan-Meier curve of overall survival analysis according to hypothyroidie as a sunitinib adverse affect

$$
\begin{aligned}
& \neg \text { hypothyroidie } \\
& { }^{r}=\text { normal thyroid function }
\end{aligned}
$$

Fig. 3. The patients with hypothyroidism as a sunitinib side effect had significantly longer OS and PFS than the patients without hypothyroidism ( $p=0.004$ and $p=0.02$, respectively)

observed. The incidence rates of the most common adverse events requiring dose reduction, such as mucositis and fatigue, were considered higher rates than reported in previous trials by Motzer et al. and Demetri et al. [2, 22]. In this patient population, we observed a lower incidence of thrombocytopenia, leukopenia and anemia than that reported in the largest trial on sunitinib so far [2]. In our trial patients with hematologic toxicity as a side ef-

\begin{tabular}{|c|c|c|c|c|}
\hline Parameter & Median OS (months) & $p$ & Median PFS & $p$ \\
\hline Sunitinib duration (longer than 1 year/shorter than 1 year) & 47 vs. 21 & $<0.0001$ & 26 vs. 16 & 0.001 \\
\hline Metastasectomy (yes/none) & 47 vs. 26 & 0.630 & 24 vs. 19 & 0.334 \\
\hline Operated primary tumour (yes/none) & 26 vs. 36 & 0.168 & 19 vs. 26 & 0.708 \\
\hline Prognostic group MSKCC (favorable/intermediate/poor) & 47 vs. 36 vs. 27 & 0.006 & 24 vs. 18 & 0.121 \\
\hline Treatment Schedule (4/2 intermittent/continuously) & 47 vs. 34 & 0.971 & 24 vs. 18 & 0.502 \\
\hline Anemia (yes/none) & 26 vs. 36 & 0.641 & 8 vs. 18 & 0.730 \\
\hline Trombocytopenia (yes/none) & 21 vs. 36 & 0.150 & 12 vs. 24 & 0.097 \\
\hline Leucocytopenia (yes/none) & 12 vs. 368 & 0.011 & 6 vs. 24 & 0.051 \\
\hline Hypertension (yes/none) & 20 vs. 27 & 0.802 & 19 vs. 18 & 0.940 \\
\hline Hypothyrodia (yes/none) & & 0.004 & & 0.020 \\
\hline Mucositis (yes/none) & 36 vs. 21 & 0.070 & 26 vs. 19 & 0.275 \\
\hline Hand foot syndrome (yes/none) & 25 vs. 36 & 0.757 & 16 vs. 24 & 0.419 \\
\hline Fatigue (yes/none) & 25 vs. 47 & 0.032 & 18 vs. 24 & 0.073 \\
\hline Rash (yes/none) & 36 vs. 25 & 0.705 & 26 vs. 16 & 0.015 \\
\hline Diarrhea (yes/none) & 21 vs. 26 & 0.287 & 11 vs. 19 & 0.407 \\
\hline Any grade 3-4 side effect (yes/none) & 12 vs. 27 & 0.281 & 6 vs. 19 & 0.108 \\
\hline
\end{tabular}
fect of sunitinib did not have significantly longer OS and PFS. It was found that leukopenia could predict survival as

Table 1. OS and PFS analyses of prognostic factors 
Table 2. Patients characteristics according two different treatment protocol

\begin{tabular}{|c|c|c|c|c|c|c|c|c|c|c|c|}
\hline Parameter & \multicolumn{5}{|c|}{$\begin{array}{c}4 \text { weeks on } 2 \text { weeks of } \\
\text { protocol }\end{array}$} & \multicolumn{4}{|c|}{ Continouous protocol } & & $p$ \\
\hline Gender (women/men) & \multicolumn{5}{|c|}{$(24 / 10)$} & \multicolumn{5}{|c|}{$(12 / 24)$} & 0.02 \\
\hline No: of metastatic lesion & 1 & 2 & 3 & 4 & 5 & 1 & 2 & 3 & 4 & 5 & 0.441 \\
\hline No: of patients & 16 & 10 & 8 & 1 & 1 & 8 & 14 & 10 & 1 & 1 & \\
\hline Metastasectomy (+/-) & \multicolumn{5}{|c|}{$(8 / 28)$} & \multicolumn{5}{|c|}{$(10 / 24)$} & 0.460 \\
\hline Nephrectomy (+/-) & \multicolumn{5}{|c|}{$(36 / 36)$} & \multicolumn{5}{|c|}{$(28 / 6)$} & 0.047 \\
\hline Prognostic group (MSKCC) (good/intermediate/poor) & \multicolumn{5}{|c|}{$(2 / 24 / 10)$} & \multicolumn{5}{|c|}{$(2 / 26 / 6)$} & 0.599 \\
\hline Age (older 65/65 and younger) & \multicolumn{5}{|c|}{$(12 / 24)$} & \multicolumn{5}{|c|}{$(16 / 18)$} & 0.241 \\
\hline Sutent dose reduction (+/-) & \multicolumn{5}{|c|}{$(30 / 6)$} & \multicolumn{5}{|c|}{$(28 / 6)$} & 0,913 \\
\hline Mortality (death/alive) & \multicolumn{5}{|c|}{$(14 / 22)$} & \multicolumn{5}{|c|}{$(18 / 16)$} & 0.505 \\
\hline
\end{tabular}

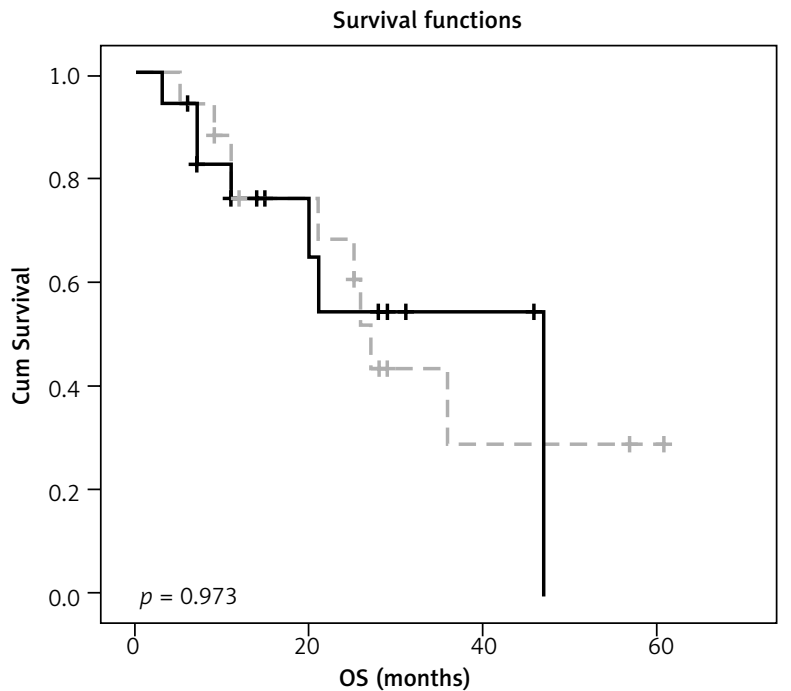

Kaplan-Meier curve of overall survival analysis according to sunitinib schema $\neg$ 4/2 intermittent

$r-$ continuous

Fig. 4. There is no significant difference between two sunitinib schema regarding OS (4/2 intermittent vs. continuous) an adverse effect of sunitinib [20]. But patients who had higher baseline leukocyte levels could have greater leukocyte reduction with sunitinib treatment [20]. The results in the literature are conflicting, and still there is no decisive information whether baseline leukopenia is a prognostic factor $[23,24]$. Hence, we need further randomized trials to clarify whether leukopenia is a predictive factor for survival, as a side effect of sunitinib. In a retrospective trial from Turkey, Dirican et al. found a higher incidence rate of anemia and thrombocytopenia compared to our study and a similar rate of leukopenia as hematologic toxicity of sunitinib. They also noted that a lower neutrophil lymphocyte rate $(<3)$ is associated with better prognosis [25]. In our study fatigue was one of the poor prognostic factors for OS. Houk Brett et al. identified tentative relationships between sunitinib exposure and fatigue, diastolic hypertension, and neutropenia. However, analysis of the adverse events was limited by the paucity of placebo data and the narrow range of doses, making it difficult to attribute these adverse events to sunitinib alone versus the disease process itself [20]. Although hypertension is probably the most studied of the toxicities, we did not find

Table 3. Number and percentage of adverse effects of two different treatment schedules

\begin{tabular}{|c|c|c|c|c|c|c|c|}
\hline \multirow[t]{2}{*}{ Parameter } & \multicolumn{3}{|c|}{4 weeks on 2 weeks of protocol } & \multicolumn{3}{|c|}{ Continouous protocol } & \multirow[t]{2}{*}{$p$} \\
\hline & $n$ & $\%$ & total & $n$ & $\%$ & total & \\
\hline Anemia & 10 & 28 & 36 & 6 & 18 & 34 & 0.313 \\
\hline Trombocytopenia & 2 & 05 & 36 & 4 & 12 & 34 & 0.354 \\
\hline Lecocytopenia & 8 & 22 & 36 & 0 & 0 & 34 & 0.003 \\
\hline Hypertension & 8 & 22 & 36 & 6 & 18 & 34 & 0.632 \\
\hline Hypothyroidie & 8 & 22 & 36 & 2 & 06 & 34 & 0.051 \\
\hline Mucositis & 18 & 50 & 36 & 18 & 53 & 34 & 0.806 \\
\hline Hand-foot syndrome & 6 & 17 & 36 & 6 & 18 & 34 & 0.913 \\
\hline Dermatologic toxicity & 10 & 28 & 36 & 14 & 41 & 34 & 0.238 \\
\hline Fatigue & 28 & 78 & 36 & 22 & 65 & 34 & 0.226 \\
\hline Diarrhhea & 6 & 28 & 36 & 2 & 06 & 34 & 0.156 \\
\hline Dose reduction & 30 & 83 & 36 & 28 & 82 & 34 & 0.913 \\
\hline Sutent duration (longer than 1 year) & 10 & 28 & 36 & 10 & 29 & 34 & 0.880 \\
\hline Any grade $3-4$ & 10 & 28 & 36 & 4 & 12 & 34 & 0.094 \\
\hline
\end{tabular}


that hypertension correlates with a better outcome as a sunitinib toxicity. Most of the published data show that grade 3-4 hypertension toxicity with sunitinib predicts good outcomes. In contrast, there was no grade 3-4 hypertensive toxicity in our study. The percentage incidence of drug-induced hypertension is $25 \%$ with sunitinib (grade 1-2: 15\%, grade 3-4: 10\%). Sunitinib-induced hypertension consistently correlated with PFS, and OS benefits in subset analyses of multiple trials [8-10]. In a series of $40 \mathrm{pa}$ tients with RCC, Rixe et al. found that toxicities limited to grade 3 hypertension were associated with response and outcome in patients treated with sunitinib [9]. Rini et al. found in a retrospective pooled analysis from four studies of patients with RCC that sunitinib-associated hypertension was associated with improved clinical outcomes [10].

Additionally, the patients with dermatologic toxicity as a side effect of sunitinib had significantly longer PFS and non-significantly longer OS. As far as it is known from the previous analysis with some anti-VEGF and TKI inhibitors, dermatologic side effects are accepted as a predictive factor [26, 27].

Several retrospective studies have demonstrated a relationship between the presence of hypothyroidism and improved antitumor efficacy in patients who had advanced renal cell carcinoma during treatment with sunitinib [1116]. As a consequence of thyroid function being measured steadily, 10 patients experienced hypothyroidism as a side effect of sunitinib. We observed that the patients with hypothyroidism as a side effect of sunitinib had significantly longer median OS and PFS, so that all of them were alive at the end of the follow-up. There are several published trials supporting that hypothyroidism as a side effect of sunitinib is related to improved clinical outcome [11-16].

The median OS of the patients in the favorable and intermediate prognostic risk groups was statistically longer than that of the poor prognostic risk group, according to MSKCC, as expected.

In a retrospective study with advanced different solid tumors (gastrointestinal stromal tumors, or RCC) in patients who were receiving sunitinib, a direct relation between the steady-state concentration of total active drug in the plasma and time to disease progression (TTP) and OS was identified [20]. We also found that the patients with longer duration of sunitinib treatment had significantly longer OS. Thus, these data underline the importance of maintaining sunitinib drug levels, but do not define which dose/schedule achieves this more optimally in a given patient with RCC. The 4/2 schedule of sunitinib at a starting dose of $50 \mathrm{mg}$ daily is the approved regimen. A continuous dosing schedule of sunitinib has been previously investigated in two phase 2 trials [28, 29]. Although prone to the significant limitations of cross-trial comparisons, these studies suggested similar efficacy outcomes between the continuous and intermittent dosing schedules and comparable toxicity.

To the best of our knowledge, the recently reported trial offers the most definitive comparison of these regimens. That trial reported results of comparing the 4/2 schedule and $37.5 \mathrm{mg}$ of sunitinib given on a continuous daily-dosing schedule and showed that the overall toxicity was sim- ilar but the quality of life was greater for the 4/2 schedule, and the time to progression was superior for this schedule in multivariable analysis [30]. The median OS and PFS of patients were not statistically significantly different in terms of treatment schemas comparing the continuous vs. $4 / 2$ schedule in our trial. Continuous dosing regimens of sunitinib at $37.5 \mathrm{mg}$ have not demonstrated an improved toxicological profile. Furthermore, patients receiving continuous dosing who require a dose reduction because of adverse events may be at risk of not achieving the target plasma concentrations. Therefore, the standard of care for initial sunitinib administration remains 50 mg daily on a 4/2 schedule. For now, patient tolerance and clinical response must be used to guide adequate sunitinib dosing while minimizing toxicity [31].

Our results suggest that hypothyroidism is probably the most reliable side effect to predict the outcome of $\mathrm{mRCC}$ and it is associated with a significant improvement of OS and PFS of sunitinib-related toxicities. We consider that if the $\mathrm{mRCC}$ patients have longer duration of sunitinib therapy, they have greater survival improvement.

Finally, we did not find any difference in survival between the two different sunitinib schemes.

Despite the retrospective design, our results are compatible with the literature and need to be validated with randomized trials.

\section{The authors declare no conflict of interest.}

\section{References}

1. Pytel D, Sliwinski T, Poplawski T, Ferriola D, Majsterek I. Tyrosine kinase blockers: new hope for successful cancer therapy. Anticancer Agents Med Chem 2009; 9: 66-76.

2. Motzer RJ, Hutson TE, Tomczak P, et al. Overall survival and updated results for sunitinib compared with interferon alfa in patients with metastatic renal cell carcinoma. J Clin Oncol 2009; 27: 3584-90.

3. Gore ME, Szczylik C, Porta C, et al. Safety and efficacy of sunitinib for metastatic renal-cell carcinoma: an expanded-access trial. Lancet Oncol 2009; 10: 757-63.

4. Shepard DR, Garcia JA. Toxicity associated with the long-term use of targeted therapies in patients with advanced renal cell carcinoma. Expert Rev Anticancer Ther 2009; 9: 795-805.

5. Motzer RJ, Bacik J, Murphy BA, Russo P, Mazumdar M. Interferon-alfa as a comparative treatment for clinical trials of new therapies against advanced renal cell carcinoma. J Clin Oncol 2002; 20: 289-96.

6. Gibbs JB. Mechanism-based target identification and drug discovery in cancer research. Science 2000; 287: 1969-73.

7. Dancey JE, Dobbin KK, Groshen S, et al. Guidelines for the development and incorporation of biomarker studies in early clinical trials of novel agents. Clin Cancer Res 2010; 16: 1745-55.

8. Chen HX, Cleck JN. Adverse effects of anticancer agents that target the VEGF pathway. Nat Rev Clin Oncol 2009; 6: 465-77.

9. Rixe O, Billemont B, Izzedine $\mathrm{H}$. Hypertension as predictive factor of sunitinib activity. Ann Oncol 2007; 18: 1117.

10. Rini BI, Cohen DP, Lu DR, et al. Hypertension as a biomarker of efficacy, with patients with metastatic renal cell carcinoma treated with sunitinib. J Natl Cancer Inst 2011; 103: 763-73.

11. Rini BI, Tamaskar I, Shaheen P, Salas R, Garcia J, Wood L, et al. Hypothyroidism in patients with metastatic renal cell carcinoma treated with sunitinib. J Natl Cancer Inst 2007; 99: 81. 
12. Wolter P, Stefan C, Decallonne B, Dumez H, Bex M, Carmeliet P, Schoffski $P$. The clinical implications of sunitinib induced hypothy roidism. A prospective evaluation. Br J Cancer 2008; 99: 448-54.

13. Baldazzi V, Tassi R, Lapini A, Santomaggio C, Carini M, Mazzanti R. The impact of sunitinib of sunitinib-induced hypothyroidism on progression-free survival of metastatic renal cancer patients: Aprospective single-center study. Urol Oncol 2010; 30: 704-10.

14. Schmidinger M. Vogl UM, Bojic M, et al. Hypothyroidism in patients with with renal cell carcinoma: Blessing or curse? Cancer 2011; 117: 534-44.

15. Makita N, Miyakawa M, Fujita T, liri T. Sunitinib induces hypothyroidism with a markedly reduced vascularity. Thyroid 2010; 20: 323-6.

16. Shinohara N, Takahashi T, Kamishima H, Ikushima H, Sazawa A, Kanayama H. Efficacy and thyroidal effects of sunitinib in Japanese patients with metastatic renal cell carcinoma: Hypothyroidism and thyroid atrophy as potential biomarkers for sunitinib? J Clin Oncol 2009; 27: 16097.

17. Faivre S, Delbaldo C, Vera K, et al. Safety, pharmacokinetic, and antitumor activity of SU11248, a novel oral multitarget tyrosine kinase inhibitor, in patients with cancer. J Clin Oncol 2006; 24: 25-35.

18. Motzer RJ, Rini BI, Bukowski RM, et al. Sunitinib in patients with metastatic renal cell carcinoma. JAMA 2006; 295: 2516-24.

19. Motzer RJ, Michaelson MD, Redman BG, et al. Activity of SU11248, a multitargeted inhibitor of vascular endothelial growth factor receptor and platelet-derived growth factor receptor, in patients with metastatic renal cell carcinoma. J Clin Oncol 2006; 24: 16-24.

20. Houk BE, Bello CL, Poland B, Rosen LS, Demetri GD, Motzer RJ. Relationship between exposure to sunitinib and efficacy and tolerability endpoints in patients with cancer: results of a pharmacokinetic/ pharmacodynamic meta-analysis. Cancer Chemother Pharmacol 2010; 66: 357-71.

21. National Cancer Institute. Common terminology criteria for adverse events v3.0 (CTCAE). August 9, 2006. Available from: (http:// ctep.cancer.gov).

22. Demetri GD, van Oosterom AT, Garrett CR, et al. Efficacy and safety of sunitinib in patients with advanced gastrointestinal stromal tumour after failure of imatinib: a randomised controlled trial. Lancet 2006; 368: 1329-38.

23. Heng DY, Xie W, Regan MM, et al. Prognostic factors for overall survival in patients with metastatic renal cell carcinoma treated with vascular endothelial growth factor-targeted agents: results from a large, multicenter study. J Clin Oncol 2009; 27: 5794-9.

24. Motzer RJ, Escudier B, Bukowski R, et al. Prognostic factors for survival in 1059 patients treated with sunitinib for metastatic renal cell carcinoma. Br J Cancer 2013; 108: 2470-7.

25. Dirican A, Kucukzeybek Y, Erten C, et al. Prognostic and predictive value of hematologic parameters in patients with metastatic renal cell carcinoma:second line sunitinib treatment following IFN-alpha. Asian Pac J Cancer Prev 2013; 14: 2101-5.

26. Di Fiore F, Rigal O, Ménager C, Michel P, Pfister C. Severe clinical toxicities are correlated with survival in patients with advanced renal cell carcinoma treated withsunitinib and sorafenib. Br J Cancer 2011; 105: 1811-3.

27. Oken MM, Creech RH, Tormey DC, Horton J, Davis TE, Mc Fadden ET, Carbone PP. Toxicity and response criteria of the Eastern Cooperative Oncology Group. Am J Clin Oncol 1982; 5: 649-55.

28. Barrios CH, Hernandez-Barajas D, Brown MP, et al. Phase 2 trial of continuous once-daily dosing of sunitinib as firstline treatment in patients with metastatic renal cell carcinoma. Cancer 2012; 118: 1252-9.

29. Escudier B, Roigas J, Gillessen S, et al. Phase II study of sunitinib administered in a continuous once-daily dosing regimen in patients with cytokine-refractory metastatic renal cell carcinoma. J Clin Oncol 2009; 27: 4068-75.

30. Motzer RJ, Hutson TE, Olsen MR, et al. Randomized phase II of sunitinib on an intermittent versus continuous dosing schedule as first-line therapy of advanced renal cell carcinoma: J Clin Oncol 2012; 30: 1371-7.

31. Suarez C, Rini BI. Determining the Optimal Dose and Schedule of Sunitinib Some Answers, More Questions. Cancer 2012; 118: $1178-80$.

\section{Address for correspondence}

\section{Çetin Ordu}

Istanbul Bilim University

Avrupa Florence Nightingale Hospital Bedrettin Mah. Bedii Gorbon Sok. No: 1, Sishane-Beyoglu

3440 Istanbul, Turkey

e-mail: cetinordu@hotmail.com

Submitted: 10.05 .2014

Accepted: $\quad 11.09 .2014$ 